\title{
Craniofacial Langerhans Cell Histiocytosis: A Rare Case Report
}

\author{
Suriyakala M., Sreela L.S., Philips Mathew, Twinkle S. Prasad and Admaja K. Nair \\ Department of Oral Medicine and Radiology, Government Dental College, Kottayam, Kerala, India
}

\begin{abstract}
Langerhans cell histiocytosis (LCH) is a rare haematological disorder characterised by accumulation and abnormal proliferation of bone marrow-derived Langerhans cells and mature eosinophils. The clinical presentation of patients with LCH may be localised or systemic, depending on the site and the degree of involvement. LCH may involve any bone, but the most commonly involved bones are long bones, pelvis, ribs, skull, vertebrae, and facial bones. In head and neck region, frontal and parietal bones are commonly involved followed by the jaws, with a predilection for mandible. In this report, we present a case of LCH involving the craniofacial bones in a 10-year boy, who presented initially with multiple ulcerative lesions on gingiva.
\end{abstract}

Key Words: Craniofacial, Langerhans cell histiocytosis, Gingiva.

How to cite this article: M. S, L.S. S, Mathew P, Prasad TS, Nair AK. Craniofacial Langerhans Cell Histiocytosis: A Rare Case Report. J Coll Physicians Surg Pak 2021; 31(10):1239-1241.

\section{INTRODUCTION}

Langerhans cell histiocytosis ( $\mathrm{LCH}$ ) is an idiopathic haematological disorder characterised by the proliferation of specialised bone marrow-derived Langerhans cells (LCS) and mature eosinophils. ${ }^{1,2}$ Previously, LCH was described under the terminology of histiocytosis $X$ by Lichtenstein in 1953 to account for three clinical conditions which exhibited similar histological characteristics, viz. eosinophilic granuloma, Letterer-Siwe syndrome, and Hand-Schuller-Christian syndrome. The terminology of LCH was introduced due to the fact that the histiocytes of the lesions showed marked similarity to that of LCs found in normal mucosa and skin. ${ }^{3} \mathrm{LCH}$ demonstrates monoclonal proliferation, accumulation, and infiltration of histiocytes along with other immune cells which leads to tissue destruction.

When jaw bones are affected, mandible is more frequently affected than the maxilla, with most of the lesions occurring in the molar and ramus region. ${ }^{4,5}$ Destruction of lamina dura and supporting bone results in the radiographic appearance of 'floating teeth'. ${ }^{6}$

LCH has traditionally been broadly categorised into three variants mainly based on the clinical manifestations: (i) Eosinophilic granuloma is a chronic, localised, single or multiple osseous lesions without extra-skeletal involvement.

Correspondence to: Dr. Suriyakala M., Department of Oral Medicine and Radiology, Government Dental College, Kottayam, Kerala, India

E-mail: suryakala94@gmail.com

Received: November 23, 2019; Revised: January 29, 2020; Accepted: February 11, 2020

DOI: https://doi.org/10.29271/jcpsp.2021.10.1239
Most commonly affected age group is 5 to 15 years. (ii) Letterer-Siwe disease manifests as acute disseminated form with multisystem involvement, which most commonly results in hepatomegaly, splenomegaly, ganglionic hypertrophy and affects children younger than 3 years of age. (iii) Hand-Schüller-Christian disease is a chronic disseminated form characterised by a triad of symptoms including exophthalmos, diabetes insipidus and osteolytic lesions of the skull; which affects most commonly children in the age group between 2 to 10 years.

Hashimoto-Pritzker syndrome is a congenital form of $\mathrm{LCH}$, which most commonly involves scalp; and recently, the nomenclature is modified as congenital self-healing reticulohistiocytosis.

\section{CASE REPORT}

A 10-year boy presented with a painless swelling in left posterior mandible of 2 weeks duration. He also complained about pain in the left post-auricular region for past 3 days. There was no history of trauma at the site. Pain was gradual in onset, mild, intermittent and non-radiating, which aggravated on touch. No other swellings were observed in his body. Past dental history revealed that he had bleeding from gums of posterior teeth of right upper and lower jaw 3 years back. Previous dental records disclosed that he underwent biopsy of the right mandibular posterior gingiva; and histopathological diagnosis was ulcerative gingivitis. Subsequently, he was treated with analgesics, antibiotics and oral prophylaxis. His medical, family, and social histories were otherwise non-contributory. On general examination, he was poorly built and nourished. Vital signs were within normal limits.

On extra-oral examination, a solitary diffuse swelling measuring approximately $1.5 \times 1 \mathrm{~cm}$ in relation to inferior border of left body of mandible was observed. Overlying skin was normal with no secondary changes. On palpation, the swelling was non-tender, afebrile, and bony hard in consistency. Left submandibular 
lymph nodes were palpable, non-tender, firm in consistency and mobile.

On intra-oral examination, ulcerative lesions were seen in relation to premolar, molar regions of all quadrants and the floor of ulcerated areas was covered with necrotic slough and granulation tissue (Figure 1). Grade III mobility in relation to left mandibular second premolar, gingival recession with furcation involvement associated with right upper and lower first molars, were also observed. His oral hygiene status was poor.

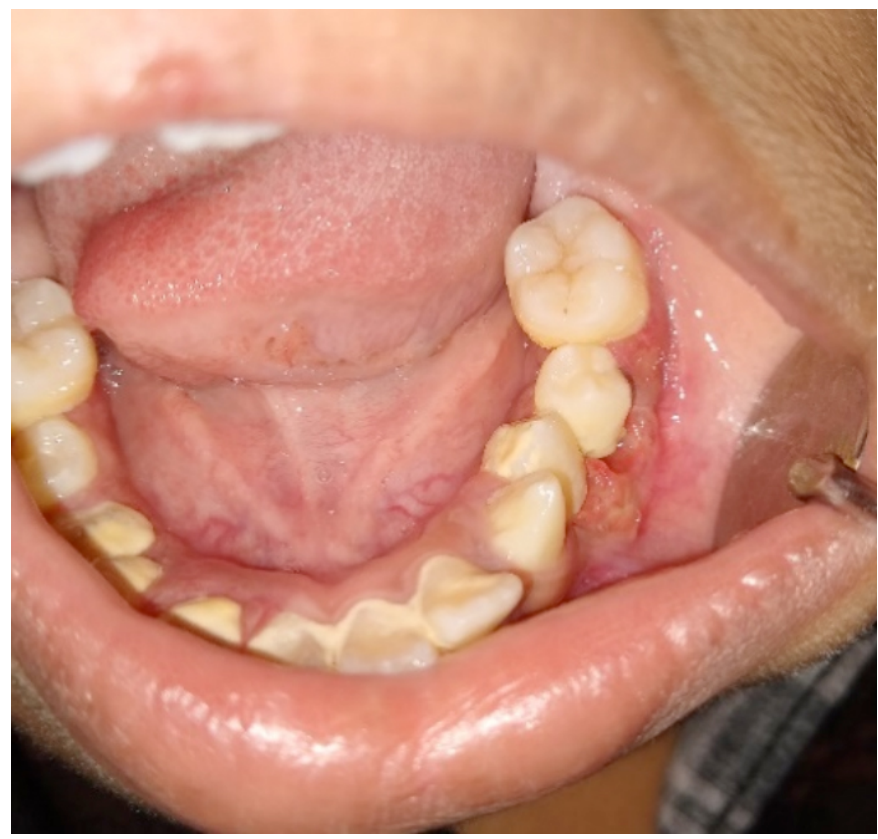

Figure 1: Intraoral view showing ulcerative gingival lesion in relation to left lowerpremolarand first molar tooth.

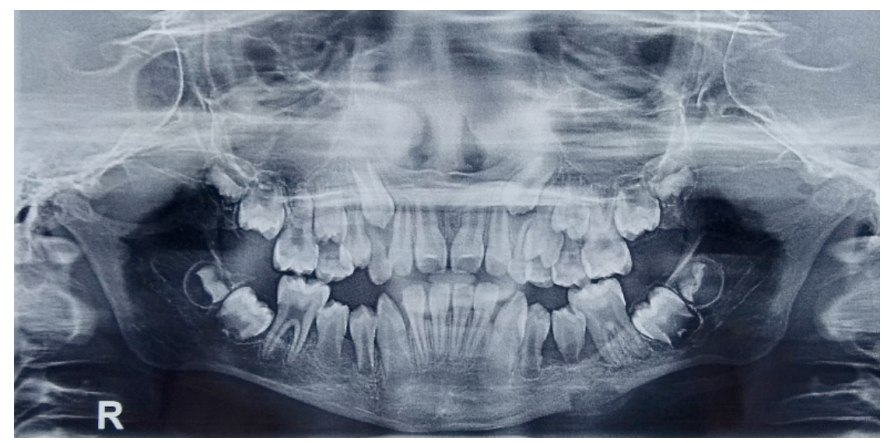

Figure 2: Panoramic radiograph showing an ill-defined osteolytic lesion in left body of mandible creating discontinuity of inferior border of mandible with periosteal reaction.

Periapical radiographs revealed localised arc shaped radiolucency in right maxillary premolar region and horizontal bone loss in left mandibular premolar region. Mandibular true occlusal view showed findings suggestive of lamellar pattern of periosteal reaction in the left premolar region. Orthopantomogram (OPG) showed an ill-defined osteolytic lesion measuring approximately $1.3 \times 0.7 \mathrm{~cm}$ in the left mandibular premolar region below the inferior alveolar canal, creating discontinuity of inferior border of mandible with periosteal reaction (Figure 2).

The differential diagnoses considered were Garre's osteomyelitis, eosinophilic granulomaandEwing'ssarcoma. Haematolog- ical investigations showed presence of mild anaemia with neutropenia and marked elevation of erythrocyte sedimentation rate (ESR). Hepatitis B surface antigen ( $\mathrm{HbsAg}$ ) and tests for human immunodeficiency virus (HIV) were negative. Ultrasound scan of abdomen did not show features of organomegaly.

MRI brain revealed lytic area measuring $7 \times 8 \mathrm{~mm}$ in the left parietal bone with beveled edges. It appeared as hyperintense in T2weighted and isointense in T1-weighted images. Another small Iytic area was noted in frontal bone. Lytic areas with indistinct air cells were noted in the left mastoid bone extending into the petrous and squamous part of temporal bone(Figure 3 ).
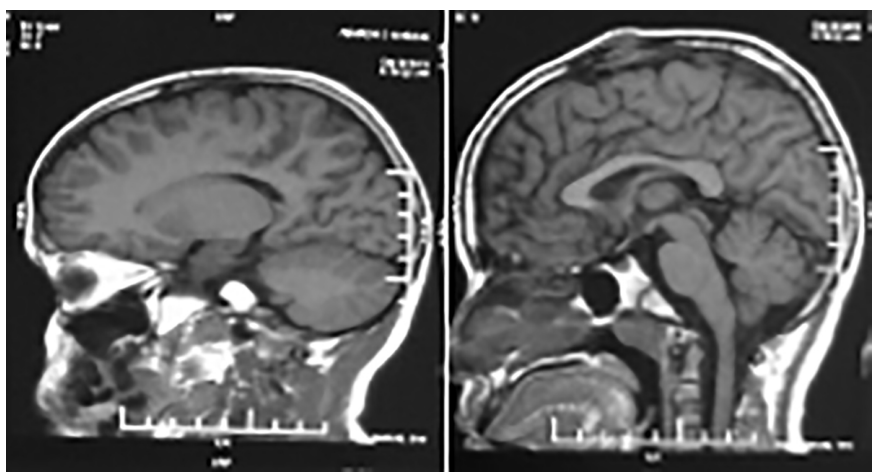

Figure 3: MRI scan image displaying lytic area with beveled edge in left parietal bone.

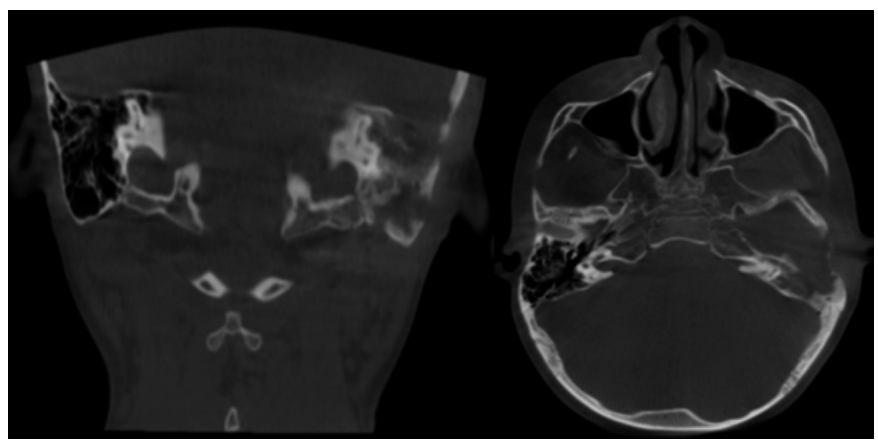

Figure 4: Coronal and axial section of CBCT image showing diffuse osteolytic lesion involving temporal bone, mastoid process and external auditorycanal.

Skeletal survey of skull displayed lytic lesions in the left high parietal calvarium and left body of the mandible. Coronal section of cone beam computed tomography (CBCT) at the level of $\mathrm{Cl}$ cervical vertebra showed diffuse osteolytic lesion involving petrous and squamous part of temporal bone, mastoid process, and superior border of external auditory canal along with loss in continuity of cortical outline. Axial section showed osteolytic lesion extending mediolaterally from lateral border of carotid canal to lateral border of temporal bone(Figure 4).

A bone biopsy was performed along with extraction of left mobile second premolar tooth. Hematoxylin and eosin stained sections revealed sheets of round and spindle shaped histiocytes. These cells showed pale stained nuclei. Cells with clear cytoplasm and eccentrically placed nuclei were evident suggestive of macrophages along with scattered plasma cells and lymphocytes. Immunohistochemistry (IHC) showed positivity for S-100, CD68, CDla and Pan-CK markers. These findings were suggestive of LCH and considering the multiple craniofacial skeletal 
involvements, patient was referred to a cancercentre forcomprehensive management.

\section{DISCUSSION}

LCH comprises of proliferation of LCs, which are dendritic mononuclear cells, usually found in the epidermis, mucosa, lymph nodes, and bone marrow. ${ }^{1}$ Even though etiology is still unclear, researchers have suggested several possible risk factors, viz. neonatal infections, especially viral infections, a skipped vaccination, environmental, genetic causes, neoplastic process and alteration in T-cell macrophage interaction. ${ }^{5}$ Papochieva et al. suggested human herpes virus 6 (HHV-6) as contributory in the modulation of $\mathrm{LCH}^{5}$ Annual incidence of $\mathrm{LCH}$ is reported as two to five cases permillion, predominantly affecting children under 15 years. ${ }^{1,6}$

Most of the reported cases were in the age group of 2 to 10 years with slight male predilection. Mandible is the second most common site in craniofacial bones after calvaria with a site predilection towards posterior mandible and ramus. In our case, osteolyic lesions involved the alveolus, inferior border of body of left-sided mandible, high parietal calvarium and left mastoid process.

Occasionally, oral manifestations are the earliest manifestations of this condition and in some cases, it may be the only manifestation of $\mathrm{LCH}$. Rao et al. observed that $5 \%-75 \%$ of patients presented with oral manifestations as earliest signs of LCH including sore mouth, gingival necrosis, mucosal ulceration, gingival hypertrophy, mobility of teeth and exfoliation, jaw pain, delayed healing of extraction sockets and localised periodontitis. ${ }^{1}$ In the present case, oral findings were gingival ulceration with necrotic slough, Grade III mobility of teeth and bony swelling of left body of mandible.

D'Ambrosio et al. summarised radiographic findings of patients with skull involvement and observed clinically asymptomatic or coexisting palpable soft-tissue mass. The lytic lesions are round or oval in shape, involving full thickness of the calvarium, with circumscribed margins, and characteristic beveled edges. Patients with temporal bone involvement typically show destructive, lytic, "punched out", bone lesions involving the mastoids, usually sparing the squamous portion and middle ear. In patients with skeletal involvement, evaluation of the extra-skeletal findings is mandatory as the treatment as well as the prognosis depends on the type and chronicity of the disorder. ${ }^{6}$ Among the types of LCH, eosinophilic granuloma shows a better prognosis. Differential diagnoses of LCH involving jaws include juvenile periodontitis, osteomyelitis, bone malignancy and metastatic tumour.

Histopathology and IHC play an important role in the diagnosis of $\mathrm{LCH}$. A definitive diagnosis of $\mathrm{LCH}$ requires that the lesional cells exhibit positive staining with S-100, CD 68 and CD1a markers in
IHC, as in our case. LCH is usually treated with surgery, radiotherapy, chemotherapy, and steroid injections, alone or in combination, depending on the extent of the disease. ${ }^{3,4}$ Importantly, all patients with $\mathrm{LCH}$ require long-term follow-up to monitor disease recurrence or other complications.

In many LCH cases, oral manifestations may be among the earliest signs of the disease, prompting patients to seek treatment. In such patients seeking dental treatment, a careful clinical examination and appropriate investigations will facilitate early diagnosis and subsequentreferral to the concerned specialists, thereby improving the prognosis.

\section{PATIENT'S CONSENT:}

Informed consents were obtained from patient's parents to publish the data.

\section{CONFLICT OF INTEREST:}

The authors declared no conflict of interest.

\section{AUTHORS' CONTRIBUTION:}

SM: Substantial contribution to the conception or design of the work.

SLS: Final approval of the version to be published.

TSP, PM, AKN: Drafting the work or revising it critically for important intellectual content.

\section{REFERENCES}

1. Rao DG, Trivedi MV, Havale R, Shrutha SP. A rare and unusual case report of langerhans cell histiocytosis. J Oral Maxillofac Pathol 2017; 21(1):140-4. doi: 10.4103/jomfp. JOMFP_10_17.

2. Luz J, Zweifel D, Hüllner $M$, Bühler $M$, Rücker $M$, Rücker B. Oral manifestation of langerhans cell histiocytosis: A case report. BMC Oral Health 2018; 18(3):106. doi: 10.1186/s12903-018-0568-5.

3. Rao S, Hegde S, Gopinath Thilak PS. Langerhans cell histiocytosis in a 5-year old girl: A case report and review of literature. Indian J Med Paediatr Oncol 2019; 40(1): S176-80. doi: 10.4103/ijmpo.ijmpo_258_17.

4. Kumar YP, Agrawal J, Mohanlakshmi J, Kumar PS. Langerhans cell histiocytosis revisited: Case report with review. Contemp Clin Dent 2015; 6(3):432-6. doi: 10.4103/0976-237X.161912.

5. Papochieva VE, Miteva DS, Perenovska PI, Petrova G. Langerhans-cell histiocytoses- epidemiology, classification, clinical features, diagnosis, complications, treatment and prognosis. J Biomed Clin Res 2016; 9(1): 3-16. doi: DOI: 10.1515/jbcr-2016-0001.

6. D’Ambrosio N, Soohoo S, Warshall C, Johnson A, Karimi S. Craniofacial and intracranial manifestations of langerhans cell histiocytosis: Report of findings in 100 patients. AJR Am J Roentgenol 2008; 191(2):589-97. doi: 10.2214/ AJR.07. 3573. 